Témoigner Témoigner. Entre histoire et mémoire

Getuigen Revue pluridisciplinaire de la Fondation Auschwitz

$125 \mid 2017$

Histoire et mémoire de la persécution des

homosexuel·le's par les nazis

\title{
Maya Zack. Installation et vidéos du cycle «Trilogie de la Mémoire »
}

Memory Trilogy (Maya Zack)

Daniel Weyssow

\section{(2) OpenEdition}

Journals

Édition électronique

URL : https://journals.openedition.org/temoigner/6114

DOI : $10.4000 /$ temoigner.6114

ISSN : 2506-6390

Traduction(s) :

Memory Trilogy (Zack) - URL : https://journals.openedition.org/temoigner/6167 [nl]

Éditeur :

Éditions du Centre d'études et de documentation Mémoire d'Auschwitz, Éditions Kimé

Édition imprimée

Date de publication : 1 octobre 2017

Pagination : 15-21

ISBN : 978-2-930953-01-4

ISSN : 2031-4183

Référence électronique

Daniel Weyssow, « Maya Zack. Installation et vidéos du cycle «Trilogie de la Mémoire » », Témoigner.

Entre histoire et mémoire [En ligne], 125 | 2017, mis en ligne le 24 décembre 2021, consulté le 04 février 2022. URL : http://journals.openedition.org/temoigner/6114 ; DOI : https://doi.org/10.4000/temoigner. 6114 


\section{MAYA ZACK. INSTALLATION ET VIDÉOS DU CYCLE "TRILOGIE DE LA MÉMOIRE "}

EXPOSITION La galerie MLF / Marie-Laure Fleisch'1 a présenté la première exposition de Maya Zack à Bruxelles du 11 novembre 2016 au 25 février 2017. Celle-ci, intitulée Memory Trilogy, réunissait les trois vidéos Mother Economy (2007), Black and White Rule (2011) et Counterlight (2016) exposées au musée d'art de Tel Aviv au printemps 2016 ainsi que l'installation Living Room (2009-2010).

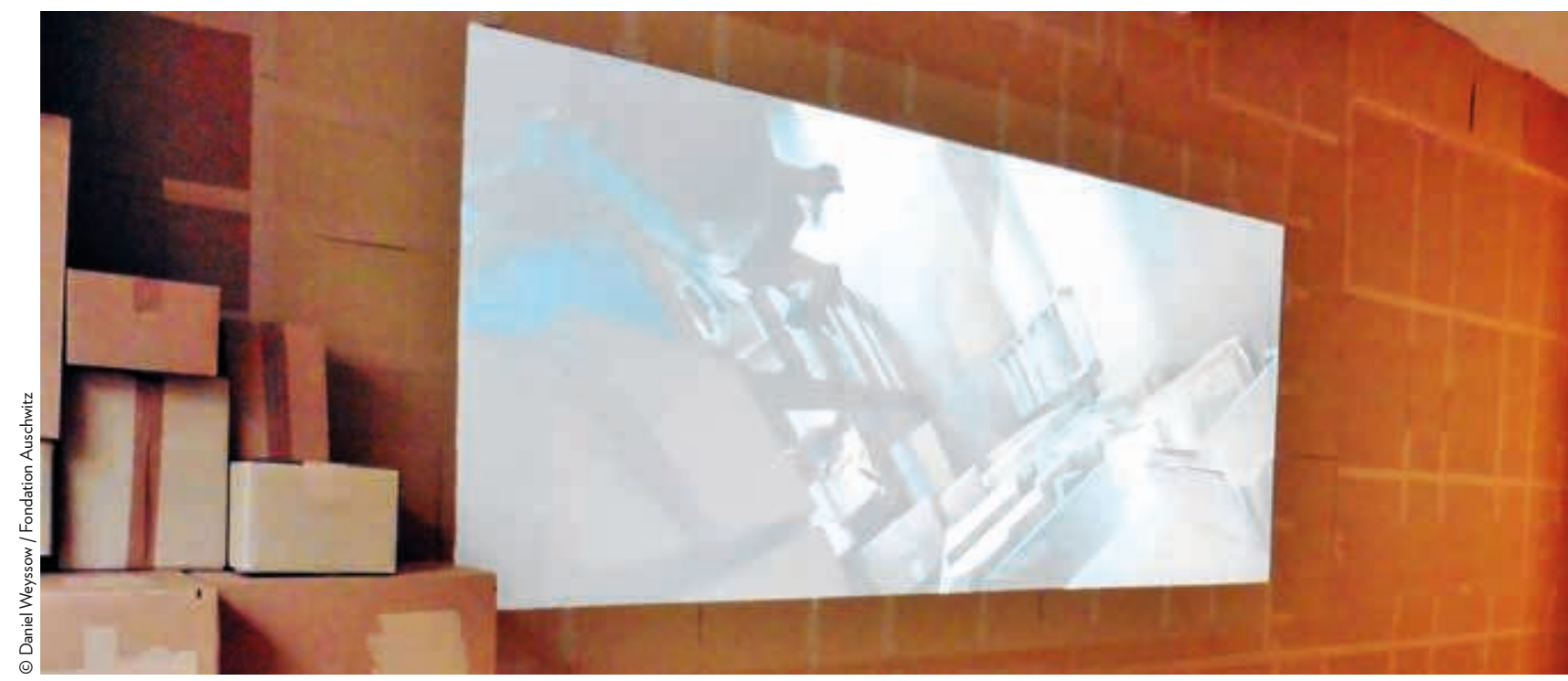

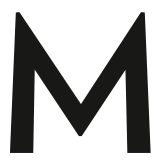
aya Zack est une artiste et réalisatrice israélienne, née en 1976 à Tel Aviv. Elle enseigne depuis 2008 à l’Académie Bezalel des Arts et Design de Jérusalem. Ses travaux ont été présentés à l'occasion de nombreuses expositions de par le monde ${ }^{2}$.

Artiste multidisciplinaire, elle allie dans un même élan photographie et cinéma, philosophie et littéra-

(1) Galerie MLF : Marie-Laure Fleisch, 13 rue Saint-Georges à 1050 Bruxelles. http://www.galleriamlf.com/artisti/15/maya-zack/

(2) Expositions personnelles : cf.www.maya-zack.com/about-contact/ ture, histoire et psychologie, arpentant le temps en interrogeant les traces et les souvenirs. Ses principales interrogations portent sur les possibilités et limites de reconnaissance et de traitement des événements passés. Serait-il envisageable d'en «ranimer» ne futce que des épisodes? Dans quelle mesure un appartement, un texte littéraire, une photo d'époque, une carte géographique, constituent-ils, éventuellement, des clés pour parvenir à se (re)saisir du passé ? Inversement, les fragments mémoriels peuvent-ils, mis en scène, soutenir la création contemporaine ? L'impossibilité de recréer l'instant passé relève de l'évidence. Photos et films visant à une telle expérience constituent néces- •.• 
- sairement un pari sur la ressemblance, aboutissant à un subterfuge, une illusion. La réalisatrice a tenté de de mém ére mises àlexamen, peuventêtreas

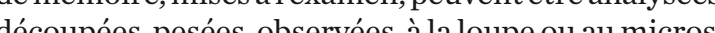

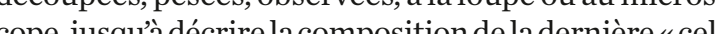
cope,jusquânécrire la compositiondeladenierencelun de nouvel Lex archives et no pas à une résurgence de a vie, à jamais éteinte.

LIVING ROOM, INSTALLATION.

IMAGES IMPRIMÉES (MODÈLE VIRTUEL 2D / 3D), EFFETS SONORES, (2009-2010)

Cette installation présente, sous cadres fixés aux murs, des modélisations concrètes développées par

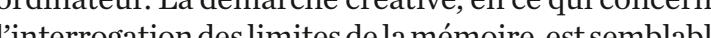
ách cycle Memory Trilogy. Il s'agit ici de la reconstitution

d'un appartement imaginée à partir des souvenirs d'un témoin qui, enfant, quitta Berlin avec ses parents en 1938 pour s'installer en Palestine. La réflexion porte sur l'environnement «type» d'une famille juive à la veille de la Shoah. Peut-on de la sorte imaginer reconstituer l'intimité d' cétic lintimite dune fanille? La mise en scène de débouche en réalité que sur la création d'un décor, qui appart de loin peut-être plus viai que nature, quis qui de loin pent-être plus mais qui est forénent inagine. De près par contre lon remarque que tout ce qui donne vie à un environnesent lexiste pas ici. Des tableaux lon ne voit que liers n'ont pas de boures. De plus, un fort sentimeliers n'ont pas de bougies. De plus, un fort sentiment dirrealite domine, les meubles semblant flotter, de perar... Lon perçoit néanmoins quils sagit avant tout de partager un inde de cement dune communauté et dune culture, même si temps.

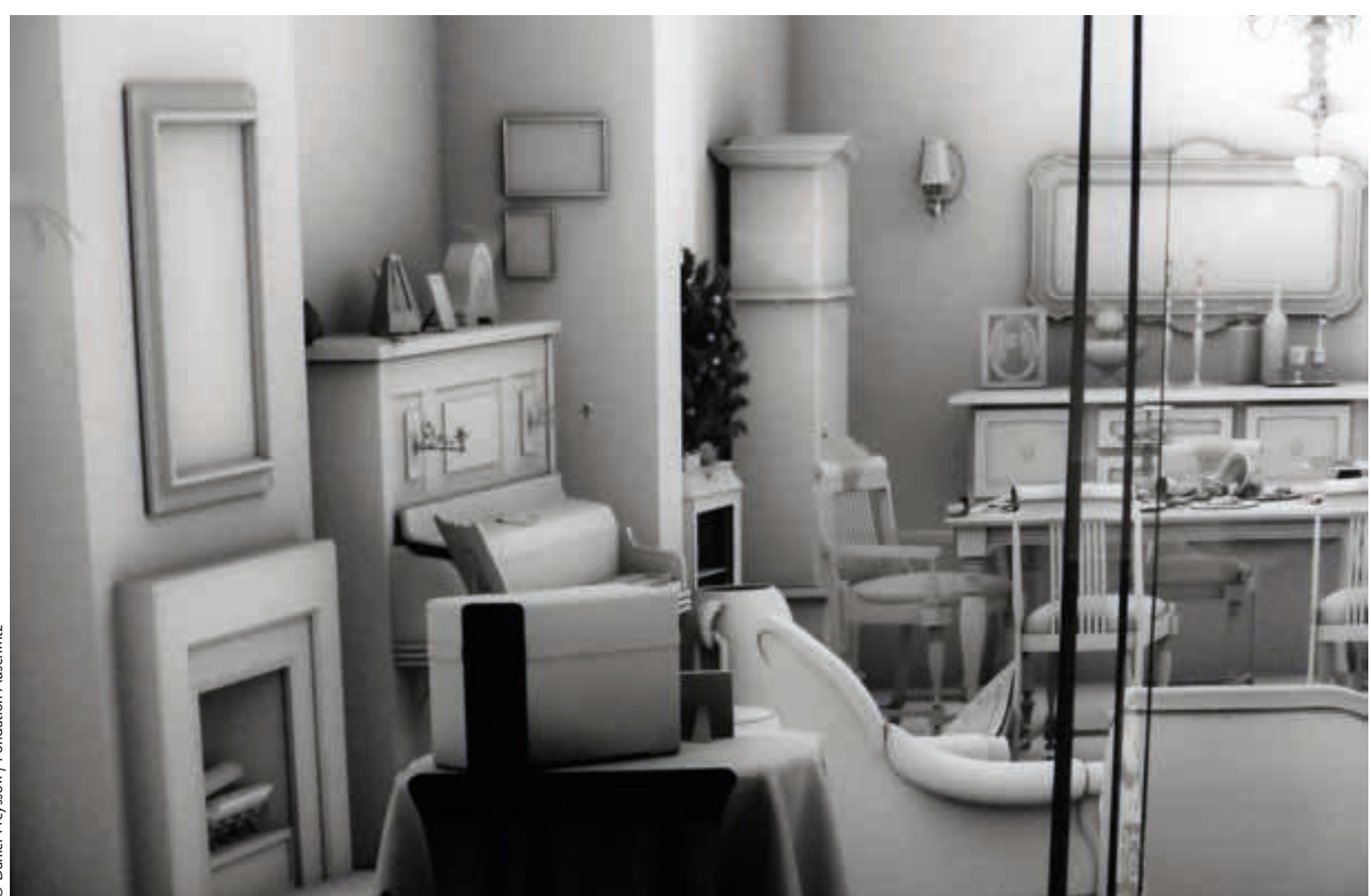

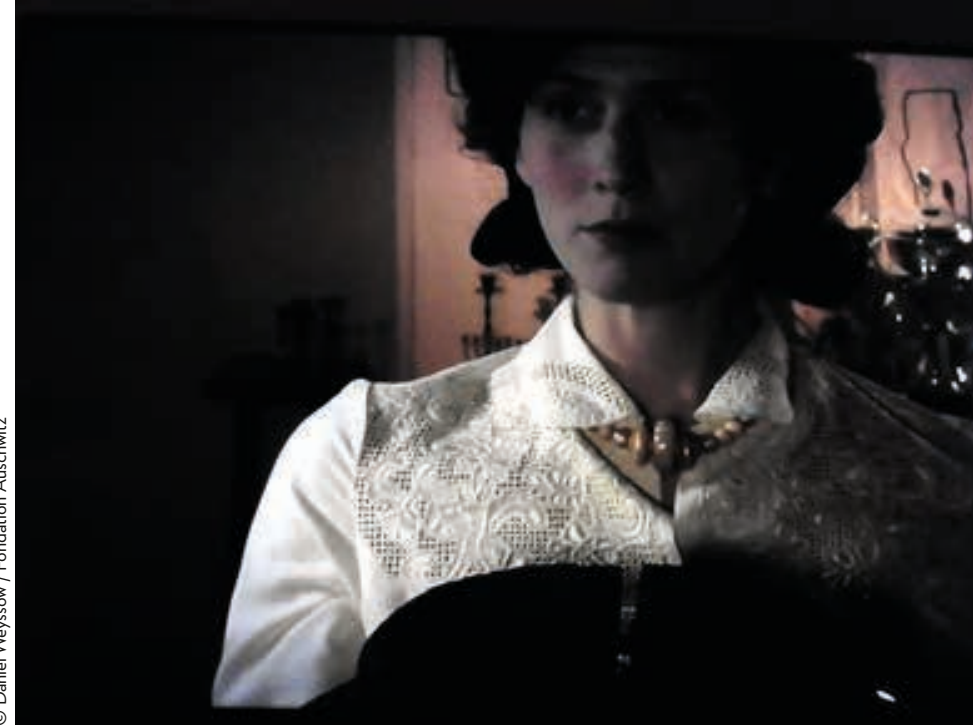

et d'autres objets personnels sur $\mathrm{d} u$ papier couvrant les murs et les planssociée aux journaux financiers ta

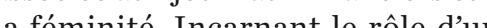
économiste, elle inventorie qui se trouvent autour delle. En utilisant un abaue et de formus con in quipernettrontcleculreun hugel (un poung aux nì̀ à quille découpera de manière àce quili ressemble a ${ }^{3}$

La réalisatrice tente de reconstituer l'appartement, qu'elle n'a pu visiter, de sa grand-mère situe en sur des objets prises de vue portent ur des on l'imanin son - on peut l'imaginer - de la rafle

La thématique de l'absence rend compte des contours du vide éprouvé, et ce par contraste aux objets «peuplant» l'appartement. L'œuvre de Maya Zack a pour conséquence de sensibiliser le spectateur aux caprices de lhistoire, a moins qu ill ne se fige, hypnotisé par le rendu hyper-détaillé et clinquant de en état de mort clinique, glacé ne peut êtrén prenté

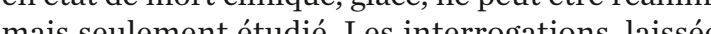
mais senlement étudie. Les interrogations, laissées l’œuvre, en raison de ce qu’elle décrit.

MOTHER ECONOMY [MĖRE ÉCONOMIE]. COURT MÉTRAGE, 2007, 19'45 MIN

Le film «est une méditation sur le souvenir de la Shoah et un hommage aux femmes ingénieuses durant Shoah et un hommage aux femmes ingenieuses durant Portint des lunettes, une blouse à lacet, pes chices. Portant des lunettes, une blouse à lacet, ses cheveux soigneusement arrangés par un chignon, la protagotâches domestiqu avec une précision etune taches dis scientifiques. La femme au foyer localise et identife

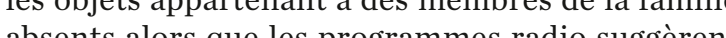
absents alors que les programmes radio suggèren l'espace quellon et chaos ont cours en dehors de tespace quelle contróle. Elle dessine une raquette de de leur restituer leurs usages. e leur restituer leurs usages. Le temps linéaire s'est

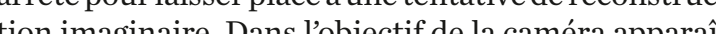
tion jug in fique, jenter fue, en blouse blanche. Interprete-t-elle le role de mimée mèredispâte du temps perdu? La reatratice pratiquer

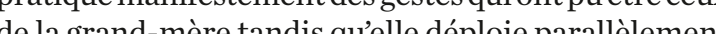
de la grand-mère tandis qu'elle déploie parallèlement maintenus dans le contexte familial dispar les objets Le film débute par la mise en persectiv

Le film debute par la mise en perspective d'u sac blanc à porter en band riliere et dun trophée de de propor ta de propagande nazie. ll faut tout deviner et se laisse emporter, rien nétant explicité. Le grand-père de une bobine de fil, des tisus. décobine de fli, des tissus. La protagoniste coud à terre. dais voci que la caméra découvre tombés nette. Un enfant? La la celle. Un méco écró gît au sol. La revis à collant. Un mégot écrasé git au sol. La revoici a terre chaises, de lable. Elle se sert d'm calier porry ins-

(3) Extrait du portfolio de l'artiste, « video / drawing / computer visualiza-
tion / installation », publie sur le site www.maya-zack.com. 
• crire chacun de ses constats. Elle classe ensuite des livres dans la bibliothèque, déplace des poussières On decouvre d'autres objets, un chandelier, un étoile juive, un pantalon d'enfant à bretelles. Elle en dispose une sur du papier millimétré et la mesure. y à aussi, qui pendent, des escarpins de ballerines. cle prend position devant un miror, se met à danRetour à par ind de tennis. Elle se relève, se remet Retour à la raquette de tennis. Elle se releve, se remet danser, constate qu'elle est blessée à la jambe. Elle drend une farde de documents quolle dépose sur un zire d'une farde de docu hents quelle depose sur un zinc despect ciou tion intén escar

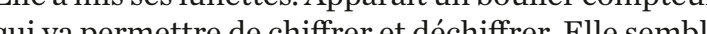
quiva per dépons, remplace dans son cahier des cher res res chiffres par des signes. Ces gribouillages restent bien

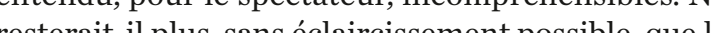
esterait-il plus, sans éclaircissent possible, que la

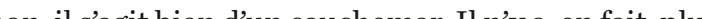

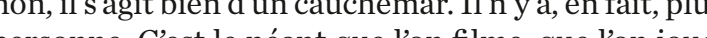
personne. Cest le neant que l'on filme, que l'on joue quelon interprète, que lon les doigts de sa min, vise. La protagoniste considère

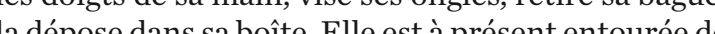
a dér. materiel de cuisine et verse de lhuile dans un enton-

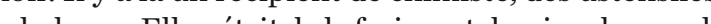
sur du p. Elle pétrit de la farine, et dessine des ronds . res. Elle y verse de leau, met quelque chose a cuire es luncttes sombunt. Ello dossine un four dont elle découpe la porte pour en sortir le gâteau cuit. Réalités imaginées et sublines se confondent. Un abaque permet d'uncula la lane dun couteau et en découpe un morceau d'un de tre de trois enfants. Elle se fige, réfléchit, dresse la table pour cinq. Le gâteau est placé au centre de la table entre

L’absence se révèle ici par la mise en place d'un processus de reconnaissance du quotidien au traver de la mise en abîme des actions et des objets servant la pise en scène. L' «écart au passén tente dêtre comblé par le travail de prospection qu'exerce la protagoniste. du fait que le passé n’a plus pour horizon qu’un ciel invisibles, absentes à jamais.

COUNTERLIGHT (2016) [CONTRE-LUMIÈRE], COURT MÉTRAGE, 23'30 MIN.

Ce film, comme le précédent, constitue un «voyage dans les profondeurs de la conscience qui suit les traces u poète, Paul Celan. Un chercheur féminin d'archives écoute des extraits d'enregistrements originaux du poète et passe du rôle d'archiviste a celui d'alchimiste Elle dissout de manière presque chirurgicale les documents darchives - photographies, cartes, documents et poèmes - qui lui permettront de reconstruire le passé lité tité lenes. Dans un processure dan lespace dune viellle photo de rue de Czernowitz et rencontre la mère de Celan qui fait cuire le pain challah dans sa cuisine, jusqua ce que sa tâche se transforme en magie féning mén monde et le processus creatif de Paul Celan, a traver les personnages feminins de sa vie, parmi lesquels sa mère, qui a été assassinée dans un camp de concente et de naissance et a consacré le film à la mémoire de

Le film débute par la vue d'une carrière exploitée à ciel ouvert. On se retrouve ensuite dans une pièce tenant à la fois de bureau, d'atelier, de salle d'archives et de clinique. La protagoniste se démène au milieu de paquets de journaux ficelés, d'archives, de chausVoicidest quinestion de lécrivain poète Paul Celan. Voici des bobines de films, un enregistreur Revox, des papiers translucides sur lesquels apparait son poème Todesfugue (1945), [Fugue de mort], scotché sur une table. Des voix de femmes en donnent des extraits. Marile. Projection sur un mur de portraits de famille. Mains gantées, la protagoniste manipule des enveloppes contenant des documents mais égalemen des cendres. Il en tombe sur la table de lecture. Elle y trace, d'un doigt, des lettres. Elle découpe le poème à

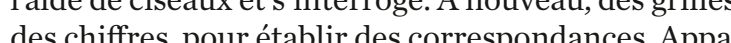
shiffres, pour établir des correspondances. Appa-

(4) Idem (traduction de l'auteur) raissent un tableau synoptique, une carte de Roumanie, des dossiers disposés sur une table en zinc, un celche d'une ville de l'enpre, pour qu'apparaissent les rues d'une ville de lempire austro-hongrois, Czernowitz qu'elle encercle dun trait - c'est celle où est né Celan. Uncoffre est ouvert, quicontient desphotos de famille, de Celan qui dit tout de la lisp lecture du poèn de Celan, qui dit tout de la «disparition». Peut-o reconnatte les personnesphotographiées? Un mètre flexible roule au sol. Des points sont fixés sur la carte. La protagoniste reapparat. Elle $n$ arche sur un trottoir, cest lle sur la photo. La quéte se poursuit, sur une découpe de la carte. They didn't lie there in, par

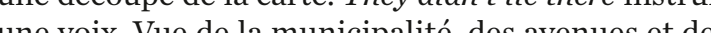
im voix. Vue de la municipanite, des avenues et des du carton. du carton. Le sealpel degage une porte. Lépaisseur duct tout doit être mélangé, revu, repensé, rémaginé dan un ordre different, car le résultat n'est pas celui que lon atten. Le Revox est renis en route. Des dossiers pentes pées, sont redisposées, certaines détourées avec une d'uningle et annotées. On aperçoit soudain, au traver dune fenetre, lintérieur dun appartement. Sur une table se détachent les mots ashes to ashes. Des poussières, descendressontranassés. La protagoniste fait leménage, d'unose s'empare d'un crayon, dépose la cendre dans un pot retroussés elle dessine sur la nap. Bras dive chemise retroussés elle dessine sur la nappe un livre ouvert de son doigt s'imp que delle y depose. L'enprente son dess(e)in, lentem son dess(e)in, lentent. La pâte prend la forme d'un cour. A proximité, le livre prend corps, composé d'un in ente

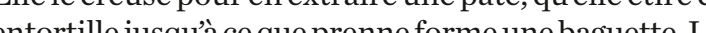
entorthlejusquáce que prenne forme une baguette. Le cour de carton et la tresse de pâte sont alors déposés sur une plaquelinéce au four. Un projecteur estallumé. livre-agonsén pulle manipule avec dou tient un rive-accor déon qu'elle rant lair ambiant. Retour aux images projetées de

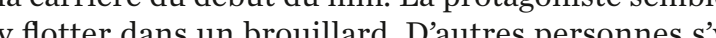
y flotter dans un brouillard. Dautres personnes sy leurs chasures sont hor Aur que ces personnes attendent leur exécution? Le livre se dégonfle alors comme un accordéon percé. Margarete d'u der un calice, d'objets en verre. La table est dressée de le livre, le cœur en papier découpé, un On y retrouve carton, le cour écon lans uposion d'un mort estmarquée à cans la positiondun mort, est marquée à terre. Laprotagoniste y dessine ce qui apparait conme son propre secting

Les œuvres de Maya Zack exposées à la galerie MLF ouvrent chacune une fenêtre sur un espace MLF ouvrent chacune une fenêtre sur un espacetranserts pre. Les situations imaginées, trufrees de réveiller et

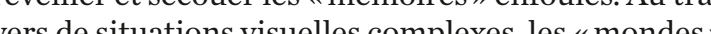
disparus quelle nou invite à explorer tou «nondes disparus quelle nous invite à explorer tournent àvic pré passé met «séquences» mises en scène servent, in fine, de Les «séqunces» nises en scène servent, in fine, de telles celles qui composent le film suivant.

\section{BLACK AND WHITE RULE (2011)}

IRĖGLANENT EN NOIR ET BLANC

VIDÉO AVEC SON, 17 MIN.

Le film « traite de la tentative humaine d'impose 'ordre et la forme sur la réalité au travers de procédures d'entraînement et de discipline. Sur un échiquier surdimensionné, deux caniches se soumetten une camera obcura Leurs action sont surveille une camera obscura. Leurs actions sont surveillees et enregistrées par ungreffier retirédans sonbureau situé leng de léch quier. Arive le moment Uniment est

Une toiletté, avance sur ses deux pattes arrière, lui donnant un aspect humain. La protagoniste observe le dresseur qui fait avancer le chien d une case a l'autre. On remarque, a teuil. Un carré de lumière éclaire un tableau. Elle y dessine le chien, ouvre un livre, y découvre une planche d'ana- ..

(5) Idem (traduction de l'auteur). 

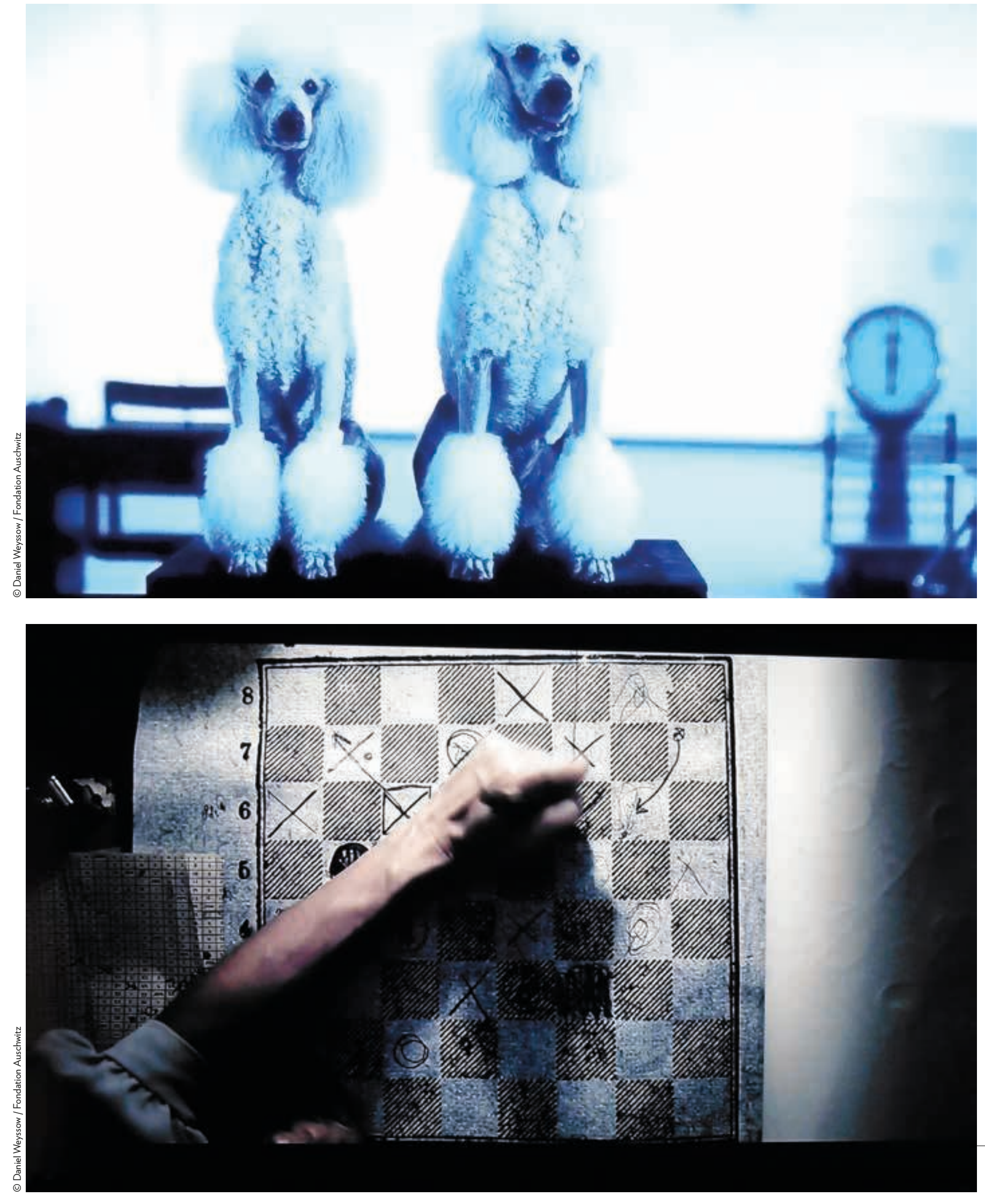

-. tomie canine. Le dresseur ausculte, prend les mesures du chien, de son crâne. Elle dessine et consigne dans son livre. Un métronome donne le ton au temps qui passe. Elle lit, puis nous fixe, nous, spectateurs, au travers d'une camera obscura, tout en manipulant une cravache. Le dresseur lance une balle de jokari. Retenue par lélastique, le caniche finit par l'attraper et recule, oujours sur ses pattes arrière, sur le damier. Tout autour, des cartons d'archives. Un projecteur entre en fonction. Et voici qu'il y a deux chiens. Le dresseur en prend un dans ses bras et l'installe sur un podium. Ils se regardent. Un coup de siflet etle chien en descend Elle tacedescercles surunéchiquien en papier $y$ inscrit les nouvelles donnés releve Sifflem chien pent doture. Le dresseur va se concher. Le chien prend egalenent place dans le sit et le regarde. bilo surl'échich

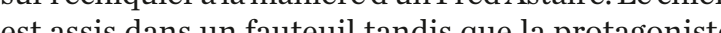
ét assit des atistion en ne saisit pas bien cequelle cher à proù on ne saisit pas bien ce quelle cherche à prouver. Des des triangles, surdes feuilles apiar. com a l'abi à labri de sa vitre, note leurs positions. Le dresseur

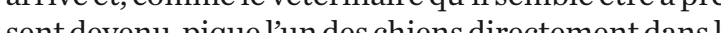
gueule Un calnu ? Non, le hien, tenu lasse au gueule. Un cal

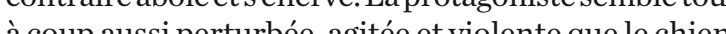
a coup aussi perture a pleur. Elle sisit unécure pleure. quenle pose sur son bureau. Lombre d'une grille suspendue projetée sur le papier sert à positionner les Mjerne paine Tout de la realisation dun dessin. Le uvérin The

Les cerves de May Zackconstituent une incita-

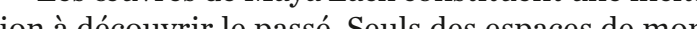
tion à découvrir le passé. Seuls des espaces de mort danslesquels circulent des fantômes, prennent forme. sein duquel se succident les thé

(6) Federico Fellinin «V Voyagea à Tulum n, épisodes publiés dans le Corriere Vincenzo Mollica « Milo Manara. Voyage à Tulum. Sur un projet de Fed scène - présence et absence, victimes et bourreaux, vie et mort -, au travers d'une archéologie des trace

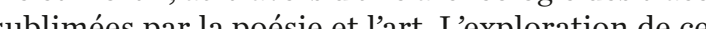
"espaces telle que la réalisatrice la pratique se déroule sous la forme de rébus d’où n’' pratique se de posibles la for uscientifiques

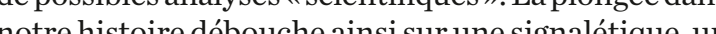
otre histoire débou che ins tenu lispar do ta pìn, des villes, des rues, des paysages, des appespoèmes, des villes, des rues, des paysages, des appardu bout du nez et que l'on excite. Ce que May Zack du tous donnibiliténjestive envers laviol ta perte de t'instendable folie des hommes dese. Le contir d'in continue dinquieter, percole au travers du rappel que t pren lâme lán en tire avoir arpentécs cesolants teritoires pour dans l'oćan de papiers sans fin dém

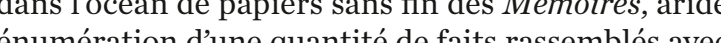
enumération dune quantité de faits rassemblés avec une rigueur statistique, in mente por l'a l'étrangé, le dégoût l'ennui était les seules vement, à mon entine dén anu sentimuent de deprinéréle

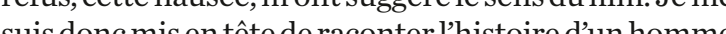
jamais jamais né, les aventures dunzon nie, funcer marionnions: [ ] p is per du ves ne se mère nù il rêve une vie sans vivre, dans un mere, sépulture d'ém rêve une vie sans vivre, dans un monde exempt pectives déclinées de facon hypnotique pectives déclinées de façon hypnotique et glacée. Ces l'envoûtement d'un aquarium, profondeur marine ou la mém dépourvu du moindre point de repè monde inconn épouru du moindre point de repère humain. Film

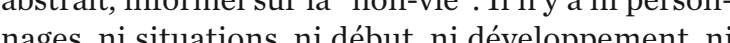
cages, ni situations, ni début, ni dévelópentiquent, but, digne d'un musée d'autón [ ] i Des duts forts, signés Federico Fellini [...].» Des mots forts, signés Federico Fellini $i^{6}$, qu situent parfaitement, appliqués aux œuvres de Maya Daniel Weyssow 